\title{
Normal cardiovascular autonomic function in patients with systemic sclerosis (CREST variant)
}

\author{
MARIA E SUAREZ-ALMAZOR, EDUARDO BRUERA, AND \\ ANTHONY S RUSSELL
}

From the Rheumatic Disease Unit, University of Alberta, 9-112 Clinical Sciences Building, Edmonton, Alberta, Canada

SUMMARY Sixteen consecutive patients with systemic sclerosis (CREST variant (calcinosis, $\stackrel{+}{ \pm}$ Raynaud's pnenomenon, oesophageal dysmotility, sclerodactyly, telangiectasia)) were included ${ }^{\infty}$ in this study. Standard non-invasive clinical and electrocardiographic procedures for the $N$ detection of cardiovascular autonomic insufficiency were performed in all patients and included o heart rate (HR) response to deep breathing, Valsalva manoeuvre, and standing, and blood pressure (BP) response to hand grip and standing. None of the patients had overt cardiovascular $\longrightarrow$ disease or were receiving cardiovascular treatment at the time of testing. Results were compared with those obtained in 17 controls. A significant difference between patients and controls was observed only in the HR and BP response to standing (results of this last procedure were within the normal expected values in both patients and controls). We conclude that patients with 9 systemic sclerosis and CREST syndrome may have an abnormal vascular response to standing; cardiovascular autonomic insufficiency, however, does not appear to be a significant feature in these patients.

Key words: scleroderma, progressive systemic sclerosis, CREST syndrome, autonomic neuropathy.

Autonomic neuropathy has been described in a variety of disorders, including diabetes mellitus, chronic renal disease, neurological disorders, advanced cancer, and rheumatoid arthritis. ${ }^{1-4}$ Several drugs, particularly those used in the treatment of cardiovascular disease, can also alter the normal autonomic response. A recent report of 14 patients suggested that autonomic dysfunction may be a feature of progressive systemic sclerosis (SS). ${ }^{5}$ We report our findings in patients with the CREST variant of SS, using standardised non-invasive methods for the diagnosis of cardiovascular autonomic insufficiency. ${ }^{1367}$

\section{Patients and methods}

Sixteen consecutive patients with SS were included in the study. Inclusion criteria were $(a)$ clinical diagnosis of SS, in compliance with the American

Accepted for publication 20 January 1988.

Correspondence to Dr Maria E Suarez-Almazor, Rhcumatic Disease Unit, University of Alberta, 9-112 Clinical Sciences Building, Edmonton, Alberta, Canada T6G 2 G3.
Rheumatism Association criteria ${ }^{\mathrm{X}}$; $(b)$ no previous history of diabetes, renal failure, arterial hypertension, or clinical cardiovascular disease; (c) no concomitant treatment with any of the following drugs: diuretics, antihypertensive or antiarrythmic drugs, digoxin, $\beta$ blockers, or adrenergic stimulants. Patients receiving calcium antagonist treatment were allowed to enter the study after discontinuation of treatment for at least 24 hours.

All the patients included in the study had the CREST variant of scleroderma." Although 23 N patients (including three with diffuse SS) had agreed to participate, seven were considered not suitable $\mathrm{N}$ (previous cardiovascular history and drug treat- $\omega$ ment) and were not included.

Cardiovascular autonomic insufficiency was as- $\stackrel{0}{C}$ sessed by clinical and electrocardiographic (ECG) tests standardised in the investigation of diabetic ? autonomic neuropathy and included $(a)$ heart rate $\overrightarrow{0}$ (HR) changes during deep breathing in the supine $\overrightarrow{\mathbb{D}}$ position, at six breaths/minute. Two measurements $\frac{?}{\mathbb{D}}$ were recorded: $(i)$ maximum $\mathrm{HR}$ variation during a $\varrho$ respiratory cycle; (ii) E:I ratio (longest RR interval 
during expiration to shortest $\mathrm{RR}$ interval during inspiration). (b) HR variation during a Valsalva manoeuvre. The 'Valsalva ratio' was calculated from the ratio of the longest $R R$ interval after the manoeuvre to the shortest RR interval during the strain. (c) HR response to standing: continuous ECG recording was performed on standing from a supine position. The ' $30 / 15$ ' ratio was calculated from the ratio of the RR intervals at beats 15 and 30 after standing. (d) Blood pressure (BP) response to standing: changes in systolic BP after standing from a supine position were recorded. (e) BP response during hand grip. Patients were asked to squeeze a blood pressure cuff at $30 \%$ of their maximal strength. Changes in diastolic BP were recorded.

Accepted abnormal values for these tests have been determined from studies in healthy populations and are as follows ${ }^{6}{ }^{7}:(a)$ maximum HR variation during deep breathing $\leqslant 10$ beats/min; $(b)$ Valsalva ratio $\leqslant 1 \cdot 10$; (c) the $30 / 15$ ratio (after standing) $\leqslant 1 \cdot 00 ;(d)$ systolic BP response to standing $\leqslant-30 \mathrm{mmHg}$; (e) diastolic BP response to hand grip $\leqslant 10 \mathrm{mmHg}$.

Seventeen healthy adults (hospital workers) were included in the study as a control population and were assessed with the same protocol. As in the SS

Table 1 Characteristics of patients and controls

\begin{tabular}{|c|c|c|c|}
\hline & Patients & Controls & $p$ Value \\
\hline $\begin{array}{l}\text { Age (years) Mean (SD) } \\
\text { Range }\end{array}$ & $\begin{array}{l}50 \cdot 4(9 \cdot 7) \\
32-63\end{array}$ & $\begin{array}{l}46 \cdot 6(9 \cdot 9) \\
33-60\end{array}$ & NS \\
\hline \multicolumn{4}{|l|}{ Sex } \\
\hline Male & 4 & 5 & NS \\
\hline Female & 12 & 12 & NS \\
\hline $\begin{array}{l}\text { Duration of Raynaud's } \\
\text { (mean (SD) years) }\end{array}$ & $14.0(12 \cdot 2)$ & - & - \\
\hline $\begin{array}{l}\text { Duration of skin sclerosis } \\
\text { (mean (SD), years) }\end{array}$ & $7 \cdot 8(6 \cdot 9)$ & - & - \\
\hline
\end{tabular}

$\mathrm{NS}=$ not significant; $\mathrm{SD}=$ standard deviation. group, none of them had previous diagnoses or concomitant treatments related to autonomic dysfunction.

Comparisons between both groups were performed with a two tailed $t$ test for unpaired data and $95 \%$ confidence intervals for the difference between the two means.

\section{Results}

Table 1 shows the characteristics of patients and controls. No significant differences were observed. Four patients were receiving nifedipine, which was discontinued at least 24 hours before entering the study. All patients had the CREST syndrome with limited skin involvement. ${ }^{9}$ Table 2 summarises the results of the autonomic function tests. A significant difference between the two groups was observed for ECG and BP response to standing only. The rest of the tests gave similar results in both groups.

\section{Discussion}

Progressive systemic sclerosis is a chronic disease with multiple organ involvement. Certain features, particularly those related to the gastrointestinal system, such as abnormal oesophageal motility, constipation, and diarrhoea, may suggest autonomic dysfunction. Non-invasive standard procedures for studying cardiac autonomic function have been developed based on the cardiovascular response to certain stimuli. ${ }^{2} 67$ These tests have shown a high correlation with autonomic neuropathy in diabetic patients $^{1}$ and have been widely used to detect autonomic insufficiency in several disorders. ${ }^{2-4} \mathrm{~A}$ recent report in 14 patients (10 CREST, four diffuse) using these methods suggested that autonomic dysfunction was a feature of SS. ${ }^{5}$ In that report, however, no information was given as to whether the patients had concomitant diseases or were receiving any treatment that might have caused

Table 2 Cardiovascular autonomic function tests. Comparison of patients with the CREST syndrome and controls

\begin{tabular}{|c|c|c|c|c|}
\hline & Patients & Controls & p Value & $\begin{array}{l}95 \% \text { Confidence } \\
\text { intervalt }\end{array}$ \\
\hline \multicolumn{5}{|l|}{ Maximum HR variation during } \\
\hline deep breathing & $16 \cdot 43(4.44) \div$ & $15 \cdot 52(5 \cdot 40)$ & NS & 4.44 to -2.62 \\
\hline $\mathrm{E}: \mathrm{I}$ ratio & $1 \cdot 27(0 \cdot 09)$ & $1 \cdot 25(() \cdot 12)$ & NS & 0.10 to $-0 \cdot() 5$ \\
\hline Valsalva ratio & $1.39(0 \cdot 20)$ & $1.41(0.34)$ & NS & 0.23 to -0.19 \\
\hline $30: 15$ ratio (standing) & $1 \cdot 00(0 \cdot 04)$ & $1 \cdot 11(0 \cdot(07)$ & $0 \cdot 0(0) 1$ & 0.15 to $0 .(07$ \\
\hline Systolic BP response to standing & $-4 \cdot 06(15.99)$ & $5.59(5.48)$ & $(0 \cdot 025$ & $18 \cdot() 4$ to 1.26 \\
\hline Diastolic BP response & $8 \cdot 50(5.49)$ & $9.47(6 \cdot 15)$ & NS & $5 \cdot 12$ to $-3 \cdot 18$ \\
\hline
\end{tabular}

*p Values not adjusted for multiplicity.

$+95 \%$ Confidence interval for the difference between the two population means.

$\ddagger$ Results are expressed as mean (SD). 
cardiovascular autonomic insufficiency. To clarify this we studied patients with SS who were free from cardiovascular treatment at the time of testing and who had no previous diagnosis related to cardiovascular autonomic insufficiency. All the patients included had the CREST syndrome with limited skin involvement ${ }^{9}$; all the patients with diffuse SS who agreed to participate were receiving treatment for hypertension or cardiovascular disease and were thus not suitable for our purpose. We were unable to find a significant difference between patients with SS and controls in most of the tests performed, and, in particular, in the HR response to deep breathing, which is considered to be the earliest and most sensitive change suggestive of autonomic dysfunction. ${ }^{6}$ A significant difference was observed only in the cardiovascular responses to standing (30: 15 ratio and BP systolic response to standing). Nevertheless, the mean BP response to standing was still in the normal range (when compared with published standard values). Furthermore, if we correct the $p$ value for the multiple comparisons made (Bonferroni's method) ${ }^{10}$ only the $30: 15$ ratio still shows a significant difference. In normal subjects systolic BP and HR increase rapidly after standing, mainly owing to an increase in sympathetic activity caused by the initial decrease in peripheral resistance. Subsequently, a relative bradycardia maximal at beat 30 is observed (parasympathetic control).${ }^{67}$ We can speculate that the difference we found in our patients when compared with controls (if true) could be due to the extensive peripheral vascular fibrosis seen in $\mathrm{SS}^{11}$; this vascular damage could cause an alteration in the normal variation in peripheral resistance owing to loss of elasticity or involvement of the terminal fibres in the vessels. The rest of the cardiac reflexes were normal; this suggests that the central nervous system autonomic control and the cardiac receptors are intact in these patients.

We cannot rule out completely the presence of cardiovascular autonomic insufficiency in SS (type IIen. error); larger numbers of patients free from drug treatment or concomitant disease would be neces? sary. This is particularly difficult in patients with $\mathrm{SS}^{\circ}$ as a significant percentage develop hypertension o흠 cardiovascular disease and receive chronic treat $\stackrel{\bar{\sigma}}{\bar{\sigma}}$ ment that may modify the autonomic reflexes.

In conclusion, we believe that patients with the CREST syndrome (when free of cardiovascular: disease) do not appear to have significant features of cardiovascular autonomic insufficiency as ascer $\vec{w}$ tained by non-invasive methods of assessing cardias reflexes. Whether patients with more extensive disease (e.g., diffuse SS) may develop cardiovascui lar autonomic insufficiency is still unknown.

\section{References}

1 Watkins P J, Mackay J D. Cardiac denervation in diabeti neuropathy. Ann Intern Med 1980; 92: 304-7.

2 Henrich W L. Autonomic insufficiency. Arch Intern Med 1982142: $339-44$.

3 Bruera E, Chadwick S, Fox R, et al. A study of cardiovascula autonomic insufficiency in advanced cancer patients. Cancép? Treat Rep 1986; 70: 1383-7.

4 Leden I, Eriksson A, Lilja B, Sturfelt G, Sundkvist G Autonomic nerve function in rheumatoid arthritis of varyingo severity. Scand J Rheumatol 1983; 12: 166-70.

5 Klimiuk P S, Taylor L, Jayson M I V. Autonomic neuropathy ip systemic sclerosis. Br J Rheumatol 1986; 25 (suppl 2): 18.

6 Hilsted J. Testing for autonomic neuropathy. Ann Clin Re 1984 16: 128-35.

7 Ewing D J, Campbell I W, Clarke B F. Assessment ơ cardiovascular effects in diabetic autonomic neuropathy an $\mathbb{P}$ prognostic implications. Ann Intern Med 1980; 92: 308-11. $\overrightarrow{\bar{O}}$

8 Subcommittee for scleroderma criteria of the Americag Rheumatism Association diagnostic and therapeutic criteria committce. Preliminary criteria for the classification of systemi sclerosis (scleroderma). Arthritis Rheum 1980; 23: 581-90.

9 Rodnan G P, Jablonska S, Medsger T A. Classification and nomenclature of progressive systemic sclerosis (scleroderma) Clin Rheum Dis 1979; 15: 5-13.

10 Ware J H, Mosteller F, Ingelfinger J A. P values. In: Bailar II J C. Mosteller F, eds. Medical uses of statistics. Massachusettsi. NEJM Books, 1986: 160-3.

11 Rodnan G P. Progressive systemic sclerosis: clinical features and pathogenesis of cutaneous involvement (scleroderma). Clig Rheum Dis 1979; 5: 49-79. 